\title{
Fully spin-polarized bulk states in ferroelectric GeTe
}

\author{
Juraj Krempaský, ${ }^{1}$ Mauro Fanciulli $\odot,{ }^{1,2, *}$ Laurent Nicolaï $\odot,{ }^{3}$ Jan Minár, ${ }^{3}$ Henrieta Volfová $\odot,{ }^{4, \dagger}$ Ondřej Caha, ${ }^{5}$ \\ Valentine V. Volobuev, ${ }^{6,7}$ Jaime Sánchez-Barriga,${ }^{8}$ Martin Gmitra, ${ }^{9}$ Koichiro Yaji, ${ }^{10}$ Kenta Kuroda,${ }^{10}$ Shik Shin, ${ }^{10}$ \\ Fumio Komori, ${ }^{10}$ Gunther Springholz, ${ }^{11}$ and J. Hugo Dil ${ }^{1,2}$ \\ ${ }^{1}$ Photon Science Division, Paul Scherrer Institut, CH-5232 Villigen, Switzerland \\ ${ }^{2}$ Institut de Physique, École Polytechnique Fédérale de Lausanne, CH-1015 Lausanne, Switzerland \\ ${ }^{3}$ New Technologies-Research Center, University of West Bohemia, Plzeň, Czech Republic \\ ${ }^{4}$ Department of Chemistry, Ludwig Maximillian University, D-81377 Munich, Germany \\ ${ }^{5}$ Department of Condensed Matter Physics, Masaryk University, Kotlářská 267/2, CZ-61137 Brno, Czech Republic \\ ${ }^{6}$ National Technical University “KhPI,” Kyrpychova Street 2, 61002 Kharkiv, Ukraine \\ ${ }^{7}$ International Research Centre MagTop, Institute of Physics, Polish Academy of Sciences, \\ Al. Lotników 32/46, PL-02-668 Warsaw, Poland \\ ${ }^{8}$ Helmholtz-Zentrum Berlin für Materialien und Energie, Elektronenspeicherring BESSY II, \\ Albert-Einstein-Straße 15, D-12489 Berlin, Germany \\ ${ }^{9}$ Institute of Physics, Park Angelinum 9, SK-040 01 Košice, Slovakia \\ ${ }^{10}$ Institute for Solid State Physics, University of Tokyo, Kashiwa, Chiba 277-8581, Japan \\ ${ }^{11}$ Institut für Halbleiter-und Festkörperphysik, Johannes Kepler Universität, A-4040 Linz, Austria
}

(Received 26 June 2019; revised manuscript received 22 November 2019; published 31 January 2020)

\begin{abstract}
By measuring the spin polarization of GeTe films as a function of light polarization we observed that the bulk states are fully spin polarized in the initial state, in strong contrast with observations for other systems with a strong spin-orbit interaction and the surface derived states in the same system. In agreement with state-of-theart theory, our experimental results show that fully spin-polarized bulk states are an intrinsic property of the ferroelectric Rashba semiconductor $\alpha$-GeTe(111). The fact that the measured spin-polarization vector does not change with light polarization can be explained by the absence of a mixing of states with a different total angular momentum $J$.
\end{abstract}

DOI: 10.1103/PhysRevResearch.2.013107

\section{INTRODUCTION}

The plethora of novel physical phenomena driven by a spin-orbit interaction (SOI) has attracted a lot of attention recently [1]. The main reason is that SOI separates charge and spin degrees of freedom and allows for spin manipulation in solids without the need for magnetic fields. However, as the name implies, SOI couples orbital and spin degrees of freedom and as a result spin is no longer a good quantum number, but systems have to be described in terms of the total angular momentum $\vec{J}=\vec{L}+\vec{S}$. This means that the "spin textures" derived for Rashba-type systems [2-7] and topological materials [8-15] do not necessarily refer to the spin quantum number $m_{s}$, but to the total angular momentum quantum number $m_{j}$ [16]. A simplified picture for this is that

\footnotetext{
"Present address: LPMS, CY Cergy Paris Université, 95031 CergyPontoise, France.

${ }^{\dagger}$ Present address: Lehrstuhl für BioMolekulare Optik, LudwigMaximilians-Universität München, Oettingenstr. 67, 80538 Munich, Germany.

Published by the American Physical Society under the terms of the Creative Commons Attribution 4.0 International license. Further distribution of this work must maintain attribution to the author(s) and the published article's title, journal citation, and DOI.
}

in a material with SOI, a state is typically made up of different orbital components and each of these orbital components has a spin component associated with it. These spin components can be parallel or antiparallel to each other and the degree of spin polarization of a state is the weighted sum of all contributions. For the example of the well-studied topological surface state of $\mathrm{Bi}_{2} \mathrm{Se}_{3}$ this leads to a degree of spin polarization of $60 \%$ [17].

Most transport applications are orbital averaging and therefore sensitive to this total spin polarization, whereas techniques based on optical transitions can be orbital selective due to the dipole selection rules. This is valid to below threshold photoexcitation as well as to photoemission. Another important property of photoemission is that even in the presence of strong SOI, spin-flip transitions are not allowed [18]. For the aforementioned surface state of $\mathrm{Bi}_{2} \mathrm{Se}_{3}$, the link between orbital and spin angular momentum is nicely illustrated by spinand angle-resolved photoemission (SARPES) experiments where the measured spin of the photoelectron reverses when the light polarization is changed and thus different orbital components are probed $[19,20]$. Recently, this concept was taken further by showing that if a linear combination of orbital components is probed, the measured spin polarization rotates in the plane perpendicular to the original direction [21]. This effect is based on spin interference and is a general property of the photoemission process $[15,22,23]$. One of the main consequences of the orbital selectivity and spin interference 
is that, also for initial states in a system with a strong spinorbit interaction, the measured degree of spin polarization will always be $100 \%$ if all three spatial components are measured and incoherent effects such as spectral broadening and background contributions are considered [15,24]. On the other hand, if the initial states are fully spin polarized with respect to the quantization axis relevant to the symmetry of the system, such so-called final state effects and spin interference, besides scattering $[25,26]$ as described in the one-step photoemission model, will no longer be relevant and the measured spin polarization will show no reversals as a function of light polarization. It will be interesting to consider to what extent (in)coherent $k_{z}$ broadening will influence the measured spin polarization for two- and three-dimensional (2D and 3D) systems, but this goes beyond the scope of this article.

The reversal of the measured spin polarization with light polarization has been predicted and detected for a variety of 2D systems with strong SOI [24,27-33] and linear dichroism in band structures has even been suggested to be a probe for spin-orbit coupling [34]. Similar selection rules have been used to reveal the hidden spin polarization in the bulk states of centrosymmetric transition metal dichalcogenides $[35,36]$. Using the additional valley degree of freedom, fully spin-polarized initial states can be realized in the related noncentrosymmetric structure of $\mathrm{MoS}_{2}$ around the $K$ points at $2 \mathrm{eV}$ binding energy [37]. However, in thin films the substrate can partly lift the symmetry protection of the valleys and the situation becomes less clear [38]. Given that most spintronic effects scale with the degree of the initial state spin polarization of bands close to the Fermi level, the question arises whether it is possible to combine a large Rashba-type spin splitting with a high $(\geqslant 95 \%)$ initial state spin polarization close to $E_{F}$ without the need for additional symmetry constraints. In this work, by combing light polarization-dependent SARPES and $a b$ initio calculations, we show that the bulk states of ferroelectric $\alpha$-GeTe fulfill these criteria, making this material a promising candidate for room-temperature spintronics applications. Our work provides evidence of fully spin-polarized initials states in any 2D or 3D system without the need for additional symmetry constraints, but based on the intrinsic orbital composition.

\section{TECHNICAL DETAILS AND ORBITAL SYMMETRY}

More than five decades ago, GeTe and SnTe were discovered to be the simplest possible displacive diatomic ferroelectrics [39]. With only two atoms per unit cell, the ferroelectric order in GeTe is caused by the relative displacement of the Ge and Te sublattice, below an ordering temperature of $700 \mathrm{~K}$. This displacement causes one of the largest observed Rashbatype spin-orbit splittings of the valence-band maximum of $4.9 \mathrm{eV} \AA$ in theory $[40,41]$ and $4.2 \mathrm{eV} \AA$ in experiment [42]. In spin pumping experiments a large spin to charge conversion was observed in $\operatorname{GeTe}(75 \mathrm{~nm}) / \mathrm{Fe}(5 \mathrm{~nm})$ stacks [43]. These results show the importance of SOI effects and hint at the functionality of $\alpha$-GeTe in this context, but the degree of spin polarization of the initial states, and thus the full potential of the material, remained unexplored.

Epitaxial films for our study of initial state spin properties were grown by molecular beam epitaxy [44] on $\mathrm{BaF}_{2}(111)$ substrates and in situ transferred for high-resolution ARPES measurements at photon energies from 11 to $26 \mathrm{eV}$ (Fig. 1), performed at the UE112-PGM2a beamline of BESSY II using $\pi$-polarized undulator radiation. Laser-based (S)ARPES experiments at $h v \approx 7 \mathrm{eV}$ were performed at the Institute for Solid State Physics, University of Tokyo, with both $\sigma$ and $\pi$ light polarization and an experimental geometry denoted in Fig. 1(d) [45]. To avoid oxidation and/or degradation, these samples had a protective stack of amorphous Te- and Se-capping layers (total thickness of $200 \mathrm{~nm}$ ). A complete removal of the protective Te/Se stack in the ultrahigh vacuum chamber was achieved by annealing at temperatures around $570 \mathrm{~K}$. The initial state band maps are presented in terms of Bloch spectral functions (BSFs) as implemented in the fully relativistic spin-polarized relativistic Korringa-KohnRostoker (SPR-KKR) theory [46].

In order to quantify the orbital composition in the initial states, Fig. 2(a) shows BSF calculations with an orbital decomposition of the Rashba-type spin-orbit splittings. Data show that for the bulk band $B_{1}$, forming the valence-band maximum, the contribution of the $p_{\frac{1}{2}}$ orbitals to the intensity and also spin polarization is negligible. To show that this absence of orbital mixing is not an artifact created by the code, we also include the full potential all-electron WIEN2K density functional code (DFT) representing the orbital character in the so-called fat bands in Fig. 2(b). It should be noted that these findings of the absence of a contribution of the $J=\frac{1}{2}$ states is in accordance with the not fully relativistic calculations by Ponet and Artyukhin [47].

\section{RESULTS}

Because the spin properties of pure surface states, surface resonance states, and bulk states are not necessarily identical [42], it is necessary to have a closer look at the measured spectral function in order to separate these different states with full confidence. Figure 1 shows ARPES data obtained on in situ transferred films with the highest resolution to date. From comparison with one-step photoemission calculations, and their general behavior as a function of photon energy $[42,48]$, we can identify three different types of bands labeled $B, S R$, and $S S$ for bulk, surface resonance, and pure surface states, respectively. All types of states show a Rashba-type spin splitting and strongly warped isoenergy cuts, in agreement with BSF theory as shown in Fig. 1(b). It can be seen how the surface resonance states are split off from the bulk states and follow these closely as a function of $k_{x}, k_{y}$, and also $k_{z}$. As a general rule of thumb, one can say that around the surface Brillouin zone (SBZ) center, the states at a slightly lower binding energy are the surface resonances, and the surface states only cross the Fermi level at significantly higher momenta.

Based on the band maps seen in Fig. 1 over a large momentum and energy range at VUV photon energies, we identify the different types of bands with high confidence in the smaller momentum and energy range available for the band structure at a photon energy of $7 \mathrm{eV}$ shown in Fig. 3. In particular, we note that within the $3 \mathrm{D} \alpha-\mathrm{GeTe}(111)$ electronic structure, ARPES data measured at $h v=7 \mathrm{eV}$ are consistent with data measured at $h v=26 \mathrm{eV}$, meaning that at $h v=7 \mathrm{eV}$ we probe 


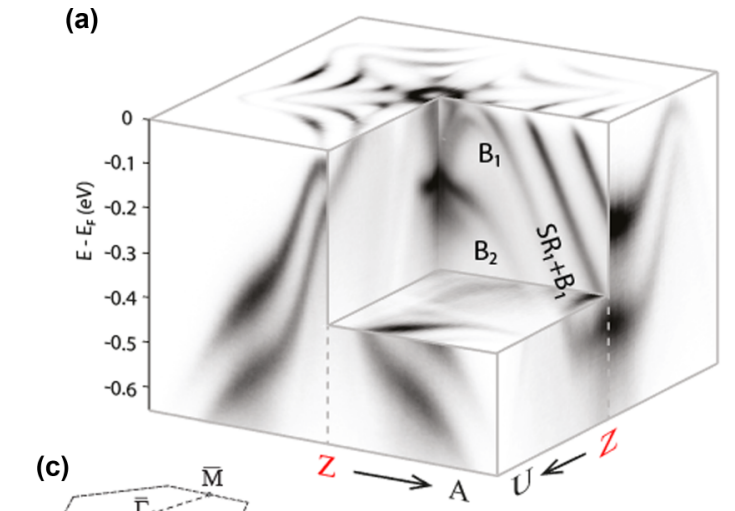

(e)
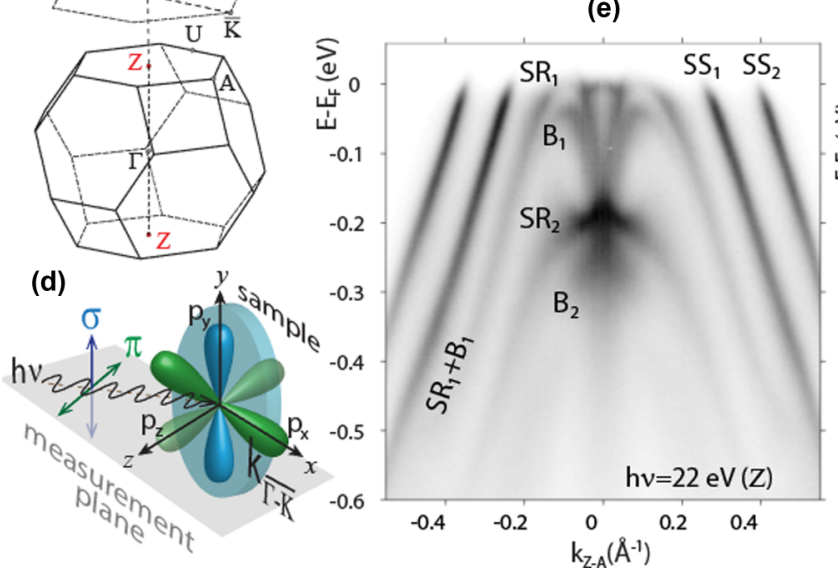

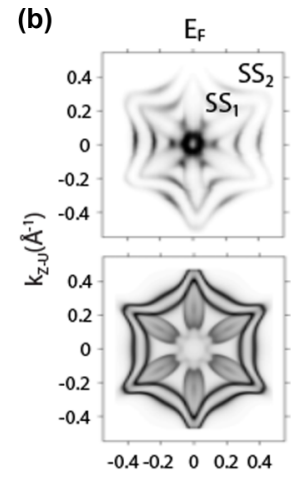

(f)
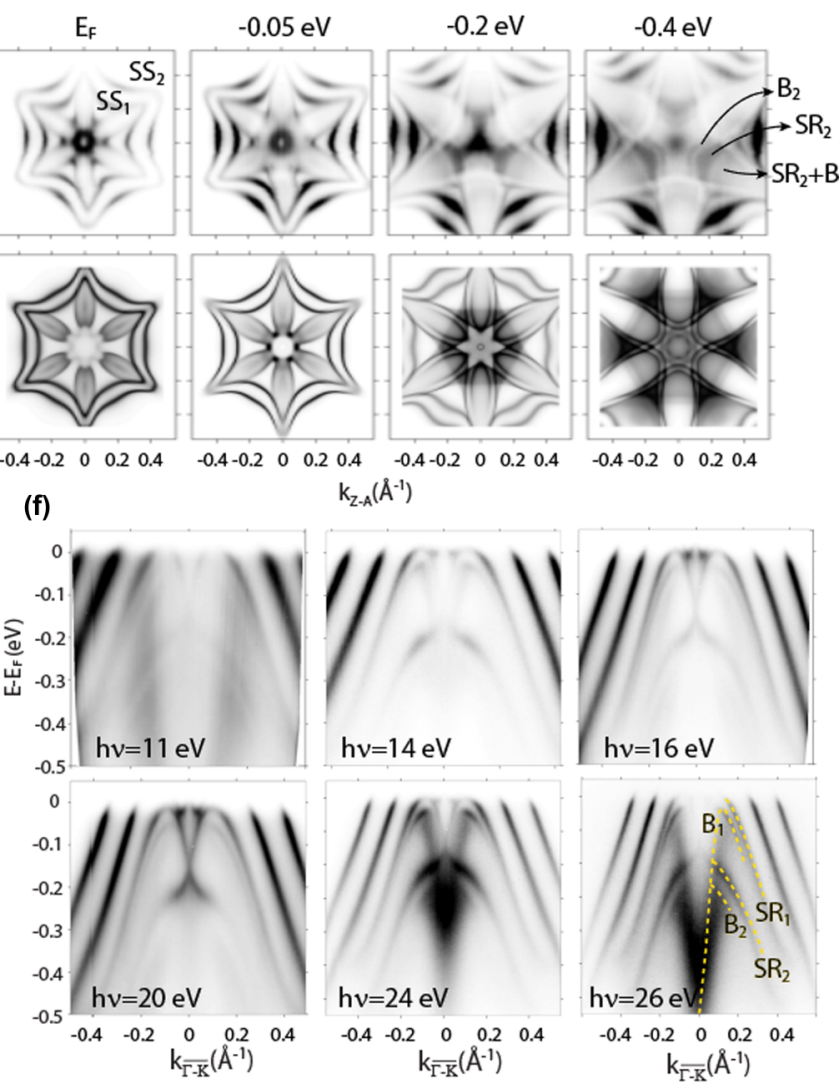

FIG. 1. (a) High-resolution $\alpha$-GeTe ARPES band structure measured around the $Z$ point $(h v=22 \mathrm{eV})$, showing the bulk Rashba-split bands $B_{1}$ and $B_{2}$, surrounded by surface resonances $S R_{1,2}$ and surface states $S S_{1,2}$. (b) Constant binding energy maps: Top panels are four selected slices from (a), and the bottom panels are corresponding BSF calculations. (c) BZ of quasicubic (rhombohedrally distorted) $\alpha$-GeTe. (d) Experimental geometry with $\pi$ - and $\sigma$-polarized light, with $\overline{K \Gamma K}$ sample alignment indicated and related $p$-orbital symmetries. (e) Representative $\alpha$-GeTe ARPES band maps at the $Z$ point $\left(h v=22 \mathrm{eV}\right.$ ). (f) ARPES band maps for selected photon energies showing the $k_{z}$ dispersion of the states. Yellow dashed lines for $h v=26 \mathrm{eV}$ serve as guides to the eye to track the different bands as used for the $h v=7 \mathrm{eV}$ data shown in Fig. 3(a).

a similar $k_{z}$ value slightly away from the $Z$ point, which is located at $h v=22 \mathrm{eV}$ [42]. As indicated by calculations of normalized $k_{z}$ values in Fig. 3(c) using an empirical inner potential $V_{0}=8.5 \mathrm{eV}$, for $h v=7 \mathrm{eV}$ we are at $k_{z} \approx 1.64 \frac{2 \pi}{c}$, where $c=5.98 \AA$ is the lattice constant of GeTe along the $z$ direction [49]. For such low photon energies the closest $Z$ point would appear around $h v=5.25 \mathrm{eV}$ and is not accessible with our laser-based (S)ARPES setup. However, as will be shown below, probing the $k_{z}$ at $h v=7 \mathrm{eV}$ does not hinder us to draw general conclusions on the fully spin-polarized initial states and is actually supporting this picture.

As illustrated in Fig. 1(d) for $\sigma$-polarized light, the orbitals with $p_{y}$ symmetry are probed, whereas for $\pi$-polarized light the orthogonal $p_{x}$ and $p_{z}$ are probed. The photoemission probabilities will depend on the overlap of the initial and final state, but from symmetry considerations the probability to probe a $p_{y}\left(p_{x, z r}\right)$ derived state with $\pi-(\sigma-)$ polarized light is zero. The data obtained with $\pi$ - [Fig. 3(a)] and $\sigma$ polarized photons [Fig. 3(b)] show the same spectral features with a slightly different intensity distribution shifted to higher momenta for $\sigma$ polarization and a higher count rate by a factor of $\approx 1.75$ for $\pi$ polarization. This indicates that although the orbital components are different, the same initial states are probed with these orthogonal light polarizations. Hence the spin polarization obtained with $\sigma$ and $\pi$ polarization can be compared directly. Our results also show that for dispersive states, linear dichroism, just as circular dichroism, is not necessarily a good probe of spin polarization.

In Figs. 4(a) and 4(b) the measured total intensity and the three spatial components of the spin polarization $\left(P_{x}, P_{y}, P_{z}\right)$ are shown for both light polarizations. The most striking observation is that the tangential, Rashba-like, component $P_{y}$ does not change with light polarization. Only the region where the surface state dominates the spectral function appears to show a polarization reversal, but the bulk states $B_{1,2}$ and the surface resonances $S R_{1,2}$ show the same $P_{y}$, only weighted differently due to the change in intensity distribution. Away from the surface states and close to the valence-band maximum also $P_{x}$ and $P_{z}$ of $B_{1}$ only show a change in distribution, but no sign change. This indicates that although orthogonal orbital components are probed, the spin vector is the same for both cases, which hints at a fully spin-polarized band. It should be noted that the sudden change in spin polarization of the states with subscript 2 in the region highlighted with a circle is related to interband or intraband spin hybridization [50-52]. 


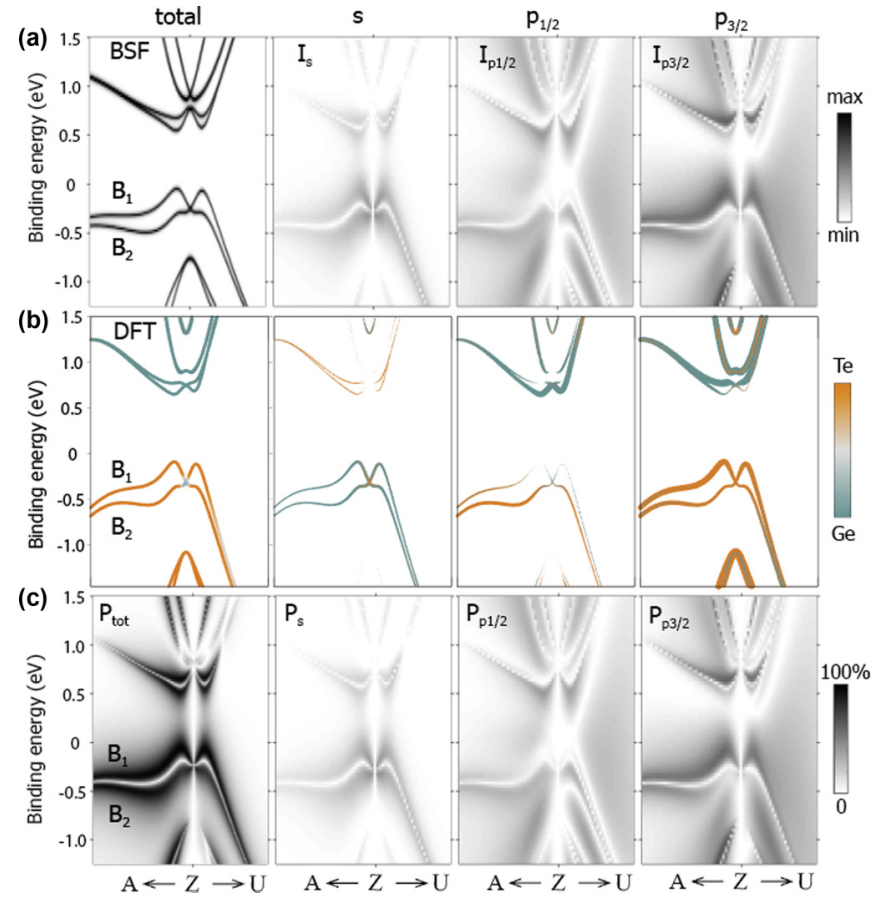

FIG. 2. (a) $\alpha$-GeTe BSF calculations with total intensity spectral function $I_{\text {tot }}$ decomposed to $s, p_{\frac{1}{2}}$, and $p_{\frac{3}{2}}$ orbital contributions; with analogous DFT analysis in (b). Color coding distinguishes the contribution from $\mathrm{Ge}$ and $\mathrm{Te}$ atoms, while circle radii represent weights of a given state projected to the corresponding relativistic orbitals, and $B_{1,2}$ are the bulk Rashba-type bands at the valence-band maximum. (c) BSF total spin-polarization initial state calculations $P_{\text {tot }}$ decomposed to individual orbitals.

\section{DISCUSSION AND COMPARISON TO THEORY}

From our SARPES experiment it is clear that the degree of initial state spin polarization of the states at the top of the valence band has to be very high and close to $100 \%$. However, our experiment does not have the geometrical flexibility to individually probe all possible orbital contributions, and furthermore, we consider only one photon energy, and thus
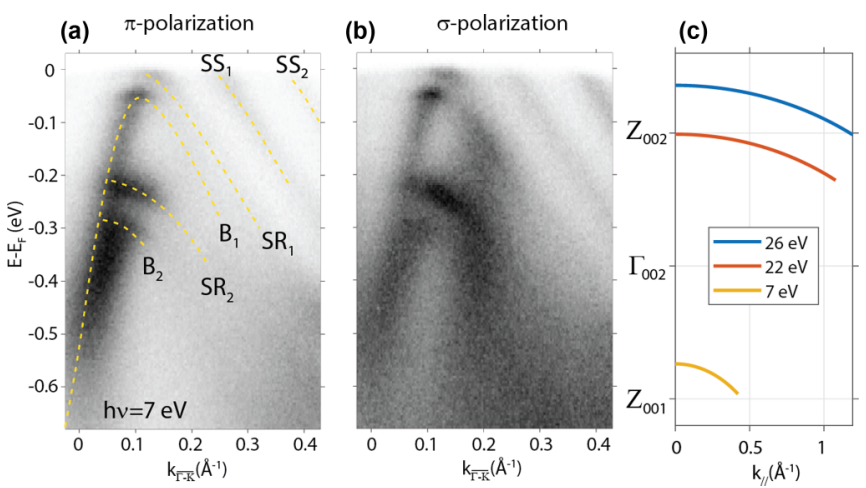

FIG. 3. ARPES band maps measured at $7 \mathrm{eV}$ for (a) $\pi$ - and (b) $\sigma$-polarized light. Dashed lines indicate the $S S_{1,2}, S R_{1,2}$, and $B_{1,2}$ bands consistent with data measured at $h v=26 \mathrm{eV}$ seen in Fig. 1(f). (c) Schematic diagram for $k$-space mapping showing normalized $k_{z}$ values based on an inner potential $V_{0}=8.5 \mathrm{eV}$. (a) $\pi$-polarization

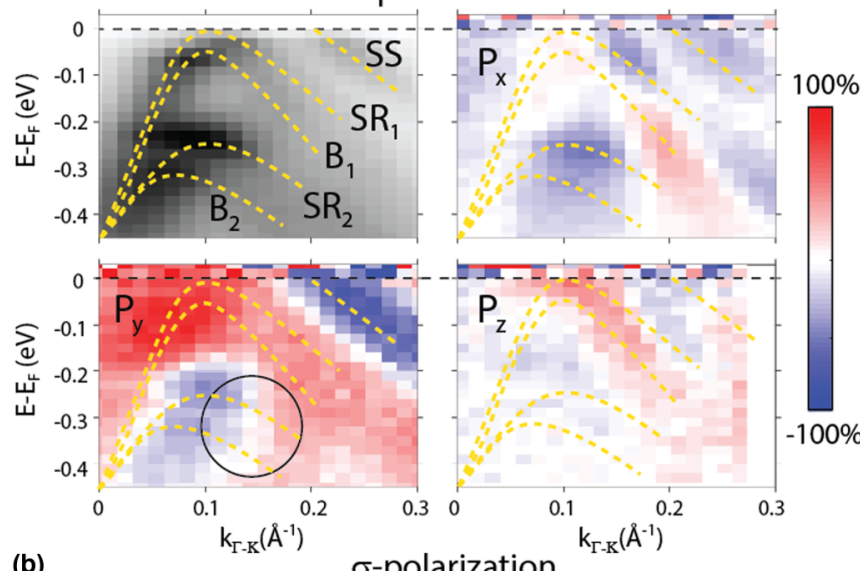

(b)
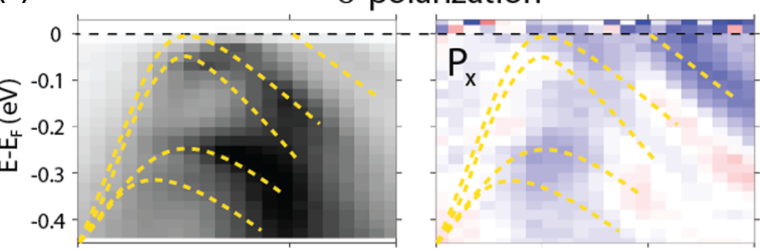

$100 \%$
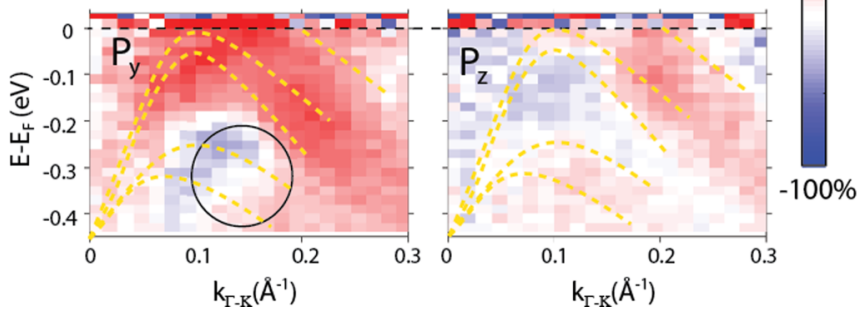

FIG. 4. (a), (b) SARPES band maps measured at $7 \mathrm{eV}$ for $\pi$ and $\sigma$-polarized light, respectively. For every panel the top left figure shows the total intensity and the other panels the respective spin polarizations along the three spatial directions. The yellow dashed lines serve as guides to the eye to track the band dispersion, and the circle highlights the region where the spin polarization changes due to spin hybridization effects.

the $k_{z}$ value for a state we know is strongly three dimensional, as shown in Fig. 1 and discussed in Ref. [42]. Therefore, to quantify this value, and study any possible dependency on $k_{z}$, we consider the initial state spectral function calculations for the bulk states shown in Fig. 5. The calculated total spin polarization in Fig. 5(a), $P_{\text {tot }}=\sqrt{P_{x}^{2}+P_{y}^{2}+P_{z}^{2}}$, is displayed along $\overline{\Gamma K}$ and $\overline{\Gamma M}$ whereby the sign is taken from the $y$ component. In the following, we only focus on the magnitude of the total degree of initial state spin polarization. In order to highlight the locus of $E(k)$ with the highest $P_{\text {tot }}$, Fig. 5(b) displays only regions where $\left|P_{\text {tot }}\right| \geqslant 0.8$. Much of the band structure remains visible, especially around the top of the valence band, the bottom of the conduction band, and two bands at a higher binding energy highlighted by the arrow. These last states were the ones studied as representative for bulk states in previous studies focused on switching the spin texture [53].

The extremely high magnitude of spin polarization at the top of the valence band becomes clear from Fig. 5(c) where $\left|P_{\text {tot }}\right| \geqslant 95 \%$. These theoretical predictions are in outstanding agreement with experimental data in Fig. 4, where almost 
(a)

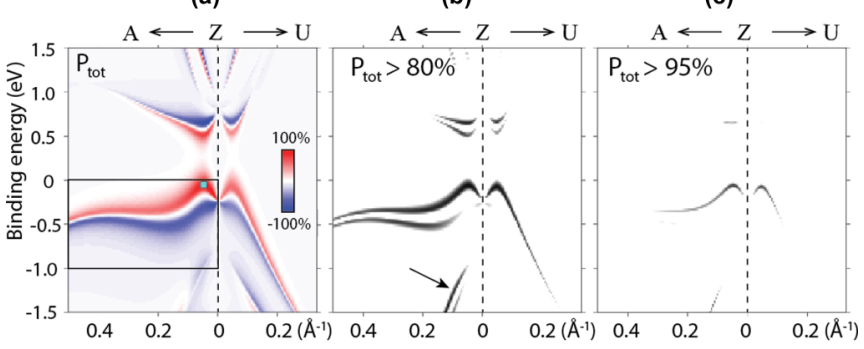

(d)
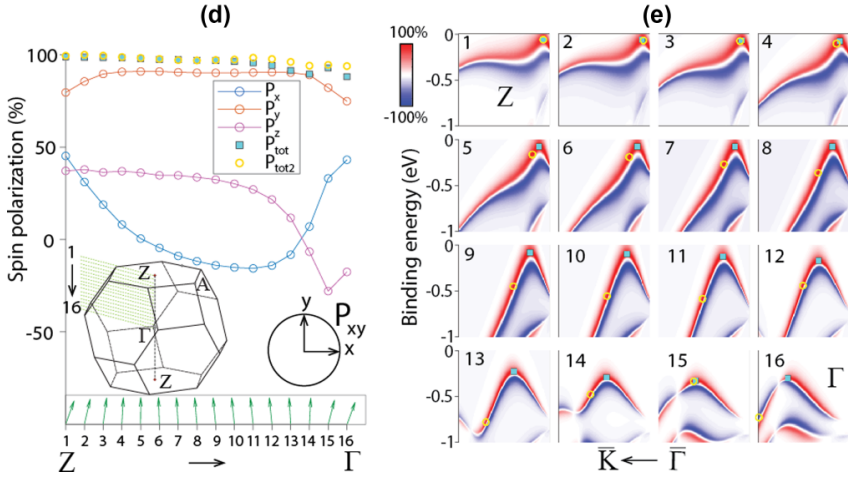

FIG. 5. (a)-(c) BSF total initial state spin-polarization calculations along the $Z-A$ and $Z-U$ directions. (a) shows total spin polarization $P_{\text {tot }}$, and the blue square indicates the locus of highest polarization. (b), (c) Band map with $\left|P_{\text {tot }}\right| \geqslant 80 \%$ and $\left|P_{\text {tot }}\right| \geqslant 95 \%$, respectively. (d) Total spin polarization $P_{\text {tot }}$ at the top of the valence band, and its spinor components $P_{x}, P_{y}$, and $P_{z}$, calculated for 16 selected momenta along the $Z \Gamma$ direction, denoted in the BZ inset. A quiver plot of their in-plane $P_{x, y}$ spin texture is seen in the bottom of the panel. For comparison, $P_{\text {tot2 }}$ indicates the maximum spin polarization found in a selected region around the top of the valence band. (e) Zoomed view [black frame in (a)] of total spin polarization along the BZ direction indicated in the inset of (d). Blue and yellow symbols show the energy and momenta where $P_{\text {tot }}$ and $P_{\text {tot2 }}$ were obtained, respectively.

pure, and identical, $P_{y}$ is observed around the valence-band maximum for both light polarizations. This large spin degree of initial state spin polarization has been found close to the Fermi level for a system with a large Rashba-type spin splitting. This is rather unexpected and follows from the examples given above, but also from studies on the bulk Rashba system $\mathrm{BiTeCl}$ [54] and the other regions in momentum space studied here for GeTe. For example, already the lower Rashba band $B_{2}$ shows a lower degree of initial state spin polarization on the ideal Rashba component, which is also reflected in the change of sign of $P_{z}$ for this state.

That this finding is not just valid for a given photon energy or $k_{z}$ value follows from the calculated $P_{x, y, z}$ and $P_{\text {tot }}$ as a function of $k_{z}$ spanning half a Brillouin zone from $\Gamma$ to $Z$ displayed in Figs. 5(d) and 5(e). Data reflect the 3D dispersion of the top of the valence band denoted by yellow circles and blue squares, with a specific canted spin texture due to a significant $P_{x}$ component close to the high-symmetry points [42], responsible for the characteristic canted in-plane $P_{x, y}$ spin texture displayed at the bottom of Fig. 5(c). The momentum and energy values for the valence-band maximum along $\Gamma Z$ are indicated by blue squares in Fig. 5(e), whereas the yellow circles are obtained by taking the maximum spin polarization within the larger momentum and energy window. Data show that the maximum calculated spin polarization is indeed closely following the dispersion of the band related to the valenceband maximum. For this reason our findings indicate that fully spin-polarized bulk states, evolving over the entire BZ from the most-pronounced Rashba-type splitting at the $Z$ point, are an intrinsic property of the $\alpha$-GeTe(111) ferroelectric Rashba semiconductor.

This raises the question on the origin of the large initial state spin polarization and whether this finding can be generalized to other systems. A well-defined initial state spin polarization requires a state with low orbital hybridization between states with different $J$, as this will also mix spin states. This can be achieved by imposing additional (valley) symmetry constraints to protect the orbital angular momentum, as is the case for $\mathrm{MoS}_{2}$ [37]. However, for GeTe, theoretical considerations show the absence of the quenching of the orbital angular momentum around the valence-band maximum and the states show a strong $J=\frac{3}{2}$ character with no contribution from $J=\frac{1}{2}$ (Fig. 2) [47]. For surface states this is rarely achieved because they either split off from bulk states or are located in an inverted band gap and thus the orbitals easily mix.

The absence of $p_{\frac{1}{2}}$ contributions to the valence-band maximum means that all orbital and thus spin components are added coherently and thus the initial state spin polarization is $100 \%$. On the other hand, the $p_{\frac{1}{2}}$ do contribute significantly to the $B_{2}$ band, and as a consequence the initial state spin polarization is predicted to be lower, as is evident from Fig. 5. The lower initial state spin polarization of this band with respect to the quantization axis relevant to the system symmetry is directly reflected in the change of its measured $P_{z}$ component as a function of light polarization shown in Fig. 4.

Combining theory and experimental results this indicates that the best strategy to look for functional high initial state spin polarization is to consider 3D states with the region close to, or crossing, the Fermi level having a pure $J$ character. The valence-band maximum of $\alpha-\mathrm{GeTe}(111)$ is a prominent example of this, but similar situations can be found in other compounds, thereby opening up the search for materials with functional high initial state spin polarization.

\section{ACKNOWLEDGMENTS}

We acknowledge the Swiss National Science foundation (SNF) Projects No. PP00P2_144742 and No. PP00P2_170591 as well as by the Austrian Science Fund (FWF), Project No. P30960-N27. J.M. and L.N. would like to thank the CEDAMNF (CZ.02.1.01/0.0/0.0/15_003/0000358) cofunded by the Ministry of Education, Youth and Sports of Czech Republic. The research of V.V.V. was supported by the Foundation for Polish Science through the IRA Programme cofinanced by EU within SG OP. J.S.-B. gratefully acknowledges financial support from the Impuls-und Vernetzungsfonds der Helmholtz-Gemeinschaft under Grant No. HRSF-0067 (Helmholtz-Russia Joint Research Group). M.G. acknowledges support by VVGS-2019-1227. 
[1] A. Soumyanarayanan, N. Reyren, A. Fert, and C. Panagopoulos, Nature (London) 539, 509 (2016).

[2] Y. A. Bychkov and E. I. Rashba, J. Phys. C: Solid State Phys. 17, 6039 (1984).

[3] M. Hochstrasser, J. G. Tobin, E. Rotenberg, and S. D. Kevan, Phys. Rev. Lett. 89, 216802 (2002).

[4] M. Hoesch, M. Muntwiler, V. N. Petrov, M. Hengsberger, L. Patthey, M. Shi, M. Falub, T. Greber, and J. Osterwalder, Phys. Rev. B 69, 241401(R) (2004).

[5] F. Meier, H. Dil, J. Lobo-Checa, L. Patthey, and J. Osterwalder, Phys. Rev. B 77, 165431 (2008).

[6] T. Hirahara, K. Miyamoto, I. Matsuda, T. Kadono, A. Kimura, T. Nagao, G. Bihlmayer, E. V. Chulkov, S. Qiao, K. Shimada, H. Namatame, M. Taniguchi, and S. Hasegawa, Phys. Rev. B 76, 153305 (2007).

[7] J. H. Dil, J. Phys.: Condens. Matter 21, 403001 (2009).

[8] M. Z. Hasan and C. L. Kane, Rev. Mod. Phys. 82, 3045 (2010).

[9] D. Hsieh, Y. Xia, L. Wray, D. Qian, A. Pal, J. H. Dil, J. Osterwalder, F. Meier, G. Bihlmayer, C. L. Kane, Y. S. Hor, R. J. Cava, and M. Z. Hasan, Science 323, 919 (2009).

[10] A. Nishide, A. A. Taskin, Y. Takeichi, T. Okuda, A. Kakizaki, T. Hirahara, K. Nakatsuji, F. Komori, Y. Ando, and I. Matsuda, Phys. Rev. B 81, 041309(R) (2010).

[11] D. Hsieh, Y. Xia, D. Qian, L. Wray, J. H. Dil, F. Meier, J. Osterwalder, L. Patthey, J. G. Checkelsky, N. P. Ong, A. V. Fedorov, H. Lin, A. Bansil, D. Grauer, Y. S. Hor, R. J. Cava, and M. Z. Hasan, Nature (London) 460, 1101 (2009).

[12] Z.-H. Pan, E. Vescovo, A. V. Fedorov, D. Gardner, Y. S. Lee, S. Chu, G. D. Gu, and T. Valla, Phys. Rev. Lett. 106, 257004 (2011).

[13] K. Miyamoto, A. Kimura, T. Okuda, H. Miyahara, K. Kuroda, H. Namatame, M. Taniguchi, S. V. Eremeev, T. V. Menshchikova, E. V. Chulkov, K. A. Kokh, and O. E. Tereshchenko, Phys. Rev. Lett. 109, 166802 (2012).

[14] G. Landolt, S. Schreyeck, S. V. Eremeev, B. Slomski, S. Muff, J. Osterwalder, E. V. Chulkov, C. Gould, G. Karczewski, K. Brunner, H. Buhmann, L. W. Molenkamp, and J. H. Dil, Phys. Rev. Lett. 112, 057601 (2014).

[15] J. H. Dil, Electron. Struct. 1, 023001 (2019).

[16] H. Zhang, C.-X. Liu, and S.-C. Zhang, Phys. Rev. Lett. 111, 066801 (2013).

[17] O. V. Yazyev, J. E. Moore, and S. G. Louie, Phys. Rev. Lett. 105, 266806 (2010).

[18] T. Y. Kim, A. Ferretti, and C.-H. Park, Phys. Rev. B 98, 245410 (2018)

[19] C. Jozwiak, C.-H. Park, K. Gotlieb, C. Hwang, D.-H. Lee, S. G. Louie, J. D. Denlinger, C. R. Rotundu, R. J. Birgeneau, Z. Hussain, and A. Lanzara, Nat. Phys. 9, 293 (2013).

[20] Y. Cao, J. A. Waugh, N. C. Plumb, T. J. Reber, S. Parham, G. Landolt, Z. Xu, A. Yang, J. Schneeloch, G. Gu, J. H. Dil, and D. S. Dessau, arXiv:1211.5998.

[21] K. Kuroda, K. Yaji, M. Nakayama, A. Harasawa, Y. Ishida, S. Watanabe, C. T. Chen, T. Kondo, F. Komori, and S. Shin, Phys. Rev. B 94, 165162 (2016).

[22] F. Meier, V. Petrov, H. Mirhosseini, L. Patthey, J. Henk, J. Osterwalder, and J. H. Dil, J. Phys.: Condens. Matter 23, 072207 (2011).

[23] U. Heinzmann and J. H. Dil, J. Phys.: Condens. Matter 24, 173001 (2012).
[24] C.-H. Park and S. G. Louie, Phys. Rev. Lett. 109, 097601 (2012).

[25] J. Kessler, Polarized Electrons, 2nd ed. (Springer, Berlin, 1985).

[26] M. Fanciulli, Spin polarization and attosecond time delay in photoemission from solids, Ph.D. thesis, École Polytechnique Fédérale de Lausanne, 2018.

[27] Z. Xie, S. He, C. Chen, Y. Feng, H. Yi, A. Liang, L. Zhao, D. Mou, J. He, Y. Peng, X. Liu, Y. Liu, G. Liu, X. Dong, L. Yu, J. Zhang, S. Zhang, Z. Wang, F. Zhang, F. Yang, Q. Peng, X. Wang, C. Chen, Z. Xu, and X. J. Zhou, Nat. Commun. 5, 3382 (2014).

[28] C. Seibel, J. Braun, H. Maaß, H. Bentmann, J. Minár, T. V. Kuznetsova, K. A. Kokh, O. E. Tereshchenko, T. Okuda, H. Ebert, and F. Reinert, Phys. Rev. B 93, 245150 (2016).

[29] H. Maaß, H. Bentmann, C. Seibel, C. Tusche, S. V. Eremeev, T. R. F. Peixoto, O. E. Tereshchenko, K. A. Kokh, E. V. Chulkov, J. Kirschner, and F. Reinert, Nat. Commun. 7, 11621 (2016).

[30] K. Miyamoto, H. Wortelen, H. Mirhosseini, T. Okuda, A. Kimura, H. Iwasawa, K. Shimada, J. Henk, and M. Donath, Phys. Rev. B 93, 161403(R) (2016).

[31] J. H. Ryoo and C.-H. Park, Phys. Rev. B 93, 085419 (2016).

[32] K. Yaji, K. Kuroda, S. Toyohisa, A. Harasawa, Y. Ishida, S. Watanabe, C. Chen, K. Kobayashi, F. Komori, and S. Shin, Nat. Commun. 8, 14588 (2017).

[33] J. H. Ryoo and C.-H. Park, Phys. Rev. B 98, 235403 (2018).

[34] H. Bentmann, H. Maaß, E. E. Krasovskii, T. R. F. Peixoto, C. Seibel, M. Leandersson, T. Balasubramanian, and F. Reinert, Phys. Rev. Lett. 119, 106401 (2017).

[35] J. M. Riley, F. Mazzola, M. Dendzik, M. Michiardi, T. Takayama, L. Bawden, C. Granerød, M. Leandersson, T. Balasubramanian, M. Hoesch, T. K. Kim, H. Takagi, W. Meevasana, P. Hofmann, M. S. Bahramy, J. W. Wells, and P. D. C. King, Nat. Phys. 10, 835 (2014).

[36] E. Razzoli, T. Jaouen, M. L. Mottas, B. Hildebrand, G. Monney, A. Pisoni, S. Muff, M. Fanciulli, N. C. Plumb, V. A. Rogalev, V. N. Strocov, J. Mesot, M. Shi, J. H. Dil, H. Beck, and P. Aebi, Phys. Rev. Lett. 118, 086402 (2017).

[37] R. Suzuki, M. Sakano, Y. J. Zhang, R. Akashi, D. Morikawa, A. Harasawa, K. Yaji, K. Kuroda, K. Miyamoto, T. Okuda, K. Ishizaka, R. Arita, and Y. Iwasa, Nat. Nanotechnol. 9, 611 (2014).

[38] S.-K. Mo, C. Hwang, Y. Zhang, M. Fanciulli, S. Muff, J. Hugo Dil, Z.-X. Shen, and Z. Hussain, J. Phys.: Condens. Matter 28, 454001 (2016).

[39] G. S. Pawley, W. Cochran, R. A. Cowley, and G. Dolling, Phys. Rev. Lett. 17, 753 (1966).

[40] S. Picozzi, Front. Phys. 2, 10 (2014).

[41] D. Di Sante, P. Barone, R. Bertacco, and S. Picozzi, Adv. Mater. 25, 509 (2013).

[42] J. Krempaský, H. Volfová, S. Muff, N. Pilet, G. Landolt, M. Radović, M. Shi, D. Kriegner, V. Holý, J. Braun, H. Ebert, F. Bisti, V. A. Rogalev, V. N. Strocov, G. Springholz, J. Minár, and J. H. Dil, Phys. Rev. B 94, 205111 (2016).

[43] C. Rinaldi, J. C. Rojas-Sánchez, R. N. Wang, Y. Fu, S. Oyarzun, L. Vila, S. Bertoli, M. Asa, L. Baldrati, M. Cantoni, J.-M. George, R. Calarco, A. Fert, and R. Bertacco, APL Mater. 4, 032501 (2016). 
[44] H. Przybylińska, G. Springholz, R. T. Lechner, M. Hassan, M. Wegscheider, W. Jantsch, and G. Bauer, Phys. Rev. Lett. 112, 047202 (2014).

[45] K. Yaji, A. Harasawa, K. Kuroda, S. Toyohisa, M. Nakayama, Y. Ishida, A. Fukushima, S. Watanabe, C. Chen, F. Komori, and S. Shin, Rev. Sci. Instrum. 87, 053111 (2016).

[46] H. Ebert, D. Ködderitzsch, and J. Minár, Rep. Prog. Phys. 74, 096501 (2011).

[47] L. Ponet and S. Artyukhin, Phys. Rev. B 98, 174102 (2018).

[48] J. Krempaský, M. Fanciulli, N. Pilet, J. Minár, W. Khan, M. Muntwiler, F. Bertran, S. Muff, A. Weber, V. Strocov, V. Volobuiev, G. Springholz, and J. Dil, J. Phys. Chem. Solids 128, 237 (2017).

[49] N. Serebryanaya, V. Blank, and V. Ivdenko, Phys. Lett. A 197, 63 (1995).
[50] H. Mirhosseini, J. Henk, A. Ernst, S. Ostanin, C.-T. Chiang, P. Yu, A. Winkelmann, and J. Kirschner, Phys. Rev. B 79, 245428 (2009).

[51] H. Bentmann, S. Abdelouahed, M. Mulazzi, J. Henk, and F. Reinert, Phys. Rev. Lett. 108, 196801 (2012).

[52] B. Slomski, G. Landolt, S. Muff, F. Meier, J. Dil, and J. Osterwalder, New J. Phys. 15, 125031 (2013).

[53] J. Krempaský, S. Muff, J. Minár, N. Pilet, M. Fanciulli, A. P. Weber, E. B. Guedes, M. Caputo, E. Müller, V. V. Volobuev, M. Gmitra, C. A. F. Vaz, V. Scagnoli, G. Springholz, and J. H. Dil, Phys. Rev. X 8, 021067 (2018).

[54] G. Landolt, S. V. Eremeev, O. E. Tereshchenko, S. Muff, K. A. Kokh, J. Osterwalder, E. V. Chulkov, and J. H. Dil, Phys. Rev. B 91, 081201(R) (2015). 